\title{
Pre-treatment Neutrophil:Lymphocyte Ratio As a Prognostic Marker in Stage III/IV Laryngeal Cancer
}

HSC) Belfast Health and
Social Care Trust $\quad$ Adams M, Ullah R

\section{Background}

Pre-treatment inflammatory cell profile may have an impact on survival in various solid cancers ${ }^{1,2}$.

\section{Objectives}

To assess the impact of pretreatment levels of circulating inflammatory cells on outcomes in stage III \& IV laryngeal cancer.

\section{Results}

Sixty-six cases of stage III/IV laryngeal cancer diagnosed in Northern Ireland between 20072010 were analysed. 39/66 (59\%) were treated with primary surgery and 27/66 (41\%) with (chemo)radiotherapy. The predictive ability of pre-treatment neutrophil:lymphocyte ration (NLR) for 5-year overall (OS) was calculated by way of a receiveroperator characteristic curve analysis and area under the curve (AOC) calculation. NLR demonstrated predictive ability for 5 year all-cause mortality for the cohort as a whole (AOC $=0.66$, 95\% Cl 0.52-0.8) (Fig 1.)

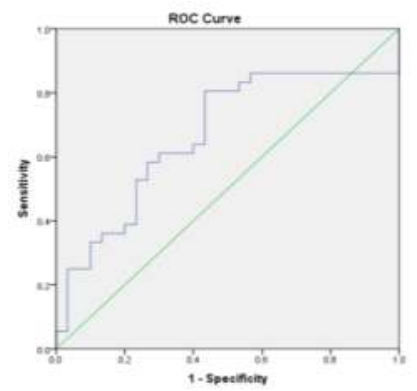

Fig 1. ROC curve for predictive ability of neutrophil:lymphocyte ratio on 5 year overall survival in stage III/IV laryngeal cancer.

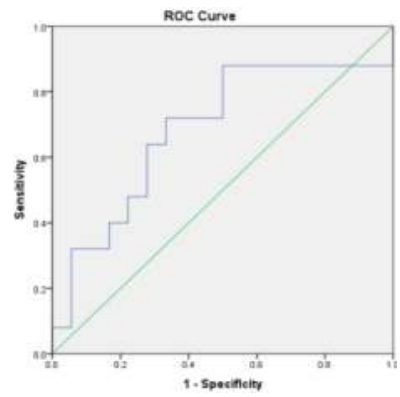

Fig 2. ROC curve for predictive ability of neutrophil:lymphocyte ratio on $5-$ year overall survival in stage III/IV laryngeal cancer treated with primary surgery.
A cut-off value of NLR $=2.81$ predicted 5-year OS with a sensitivity and specificity of $81 \%$ and $43 \%$ respectively. Further sub group analysis demonstrated that a statistically significant predictive ability was maintained in the surgical group $(\mathrm{AOC}=0.67,95 \% \mathrm{Cl}$ 0.5-0.83, Fig 2) but not the (chemo))radiotherapy group (AOC=0.61, 95\% Cl 0.37-0.86).

\section{Conclusion}

Neutrophil-lymphocyte is a sensitive but not specific predictor of survival in stage III/IV laryngeal cancer.

References

1. Nakayama et al. Modified combination of platelet count and neutrophil "to" lymphocyte ratio as a prognostic factor in patients with advanced head and neck cancer. Head \& Neck 2018;40(6):1138-1146. 